\title{
Design of Decoupled PID Controllers for MIMO Systems
}

\author{
Karl Johan Åström ${ }^{1}$, Karl Henrik Johansson ${ }^{2}$, and Qing-Guo Wang ${ }^{3}$
}

\begin{abstract}
The design of PID controllers for systems with interacting loops is discussed. It is important to deal with the interaction at the lower-level loops, since supervisory control based on for instance MPC seldom has sufficient bandwidth. A new scheme based on modified scalar PID design and static decoupling is developed, where the frequency characteristics of the coupling between the lower-level loops is taken into account. This leads to a design method emphasizing the trade-off between the individual loop performances and the so called interaction indices. The controller is easily implemented, due to its simple configuration based on standard components. The method is applied to a couple of examples.
\end{abstract}

\section{Introduction}

Model predictive control (MPC) is becoming the standard technique to solve multivariable control problems in the process industry $[12,5,8]$. Practically all MPC systems are however operating in a supervisory mode with PID controllers at the lower level. A substantial portion of the performance improvement credited to MPC is actually due to improvements in the lower-level PID loops. Interaction among the loops causes difficulties when the lower-level loops are closed. There are some difficulties in dealing with the interaction at the MPC level because the bandwidths of the MPC loops are limited: they operate in supervisory mode with sampling intervals that are longer than the PID loops. It is consequently of interest to investigate ways of dealing with interaction at the loop level [6]. A preliminary study of this problem is given in the paper. The presentation is restricted to systems with two inputs and two outputs, because such systems are common. Typical examples are boilers, machine direction moisture and basis weight control in paper machines, distillation columns, heat exchangers, and air-conditional sys-

\footnotetext{
${ }^{1}$ Department of Automatic Control, Lund University, SE22100 Lund, Sweden, kja@control.lth.se.

${ }^{2}$ Department of Signals, Sensors and Systems, Royal Institute of Technology, SE-100 44 Stockholm, Sweden, kallej@s3.kth.se. Corresponding author.

${ }^{3}$ Department of Electrical Engineering, National University of Singapore, Singapore, elewqg@nus.edu.sg.
}

tems [7]. The results can be extended to systems with more inputs and outputs.

The approach we take is to investigate standard PID tuning [2] and see what can be achieved by adding simple interactions between the feedback loops. In many cases the performance of the system can be considerably improved, particularly if the coupling in the process is not severe. The proposed scheme is based on a simple decoupling, which implies that it can be easily implemented at the loop level. The advantage by doing this is that it gives performance enhancement in a frequency range that is normally not dealt with by MPC.

The outline of the paper is as follows. The design method is described in detail in Section 2, where first the implications of decoupling is discussed in the frequency domain, followed by the introduction of a new set of interaction indices, and the design of decentralized PID controllers. In Section 3, the method is illustrated on two examples from the literature. A brief discussion is finally given in Section 4 .

\section{The Method}

Consider a multivariable control problem consisting of the design of a linear controller $C$ for a linear stable process $G$. For simplicity assume the process has two inputs and two outputs, so that $G$ is a transfer function of the form

$$
G(s)=\left(\begin{array}{ll}
g_{11}(s) & g_{12}(s) \\
g_{21}(s) & g_{22}(s)
\end{array}\right)
$$

The controller to be designed is a static decoupler combined with a decentralized PID controller with setpoint weighting. The control law can be written as

$$
\left(\begin{array}{l}
U_{1}(s) \\
U_{2}(s)
\end{array}\right)=\left(\begin{array}{ll}
d_{11} & d_{12} \\
d_{21} & d_{22}
\end{array}\right)\left(\begin{array}{l}
\bar{c}_{1}(s) Y_{r 1}(s)-c_{1}(s) Y_{1}(s) \\
\bar{c}_{2}(s) Y_{r 2}(s)-c_{2}(s) Y_{2}(s)
\end{array}\right)
$$

where $U$ is the control signal, $Y$ the process output, and $Y_{r}$ the reference. The decoupler

$$
D=\left(\begin{array}{ll}
d_{11} & d_{12} \\
d_{21} & d_{22}
\end{array}\right)
$$

is a constant matrix. The PID controller $\bar{c}_{i}$ is different from $c_{i}$ to allow for set-point weighting [2]. The 
controllers are of the form

$$
\begin{aligned}
& c_{i}=k_{P i}+\frac{k_{I i}}{s}+k_{D i} s \\
& \bar{c}_{i}=b_{i} k_{P i}+\frac{k_{I i}}{s}
\end{aligned}
$$

where $b_{i}$ is the set-point weight, which in practice often is equal to zero. Here, for simplicity we assume $b_{i}=0$ and PI control, i.e., $k_{D i}=0$. It will be shown that setting $b_{i}=0$ is essential to get good performance in decentralized PID control.

\section{Decoupling}

The static decoupler is given by

$$
D=G^{-1}(0)=\frac{1}{\operatorname{det} G(0)}\left(\begin{array}{cc}
g_{22}(0) & -g_{12}(0) \\
-g_{21}(0) & g_{11}(0)
\end{array}\right)
$$

where we have assumed that $G(0)$ is non-singular. The transfer function of the decoupled system is $Q(s)=$ $G(s) D$ where

$$
\begin{aligned}
& q_{11}(s)=\frac{g_{11}(s) g_{22}(0)-g_{12}(s) g_{21}(0)}{\operatorname{det} G(0)} \\
& q_{12}(s)=\frac{g_{12}(s) g_{11}(0)-g_{12}(0) g_{11}(s)}{\operatorname{det} G(0)} \\
& q_{21}(s)=\frac{g_{21}(s) g_{22}(0)-g_{21}(0) g_{22}(s)}{\operatorname{det} G(0)} \\
& q_{22}(s)=\frac{g_{22}(s) g_{11}(0)-g_{21}(s) g_{12}(0)}{\operatorname{det} G(0)}
\end{aligned}
$$

It follows from the construction that $Q(0)$ is the identity matrix. A Taylor series expansion of the transfer function $Q(s)$ for small $s$ gives

$$
Q(s) \approx\left(\begin{array}{cc}
1 & \kappa_{12} s \\
\kappa_{21} s & 1
\end{array}\right)
$$

for some constants $\kappa_{12}$ and $\kappa_{21}$. Hence, for low frequencies $\omega$, the diagonal elements are equal to one and the off-diagonal elements are proportional to $i \omega$. If the bandwidth of the decentralized PID controller are sufficiently low, the off-diagonal terms will thus be small and the system will be approximately decoupled.

It is straightforward to see that the closed-loop system can be described by

$$
\left(\begin{array}{cc}
1+q_{11} c_{1} & q_{12} c_{2} \\
q_{21} c_{1} & 1+q_{22} c_{2}
\end{array}\right) Y=\left(\begin{array}{cc}
q_{11} \bar{c}_{1} & q_{12} \bar{c}_{2} \\
q_{21} \bar{c}_{1} & q_{22} \bar{c}_{2}
\end{array}\right) Y_{r}
$$

where we suppressed the dependency on $s$ in the notation. This equation can be written as

$$
Y=\bar{H} Y_{r}
$$

where

$$
\begin{aligned}
\bar{h}_{11} & =\frac{q_{11} \bar{c}_{1}+\left(q_{11} q_{22}-q_{12} q_{21}\right) \bar{c}_{1} c_{2}}{\left(1+q_{11} c_{1}\right)\left(1+q_{22} c_{2}\right)-q_{12} q_{21} c_{1} c_{2}} \\
\bar{h}_{12} & =\frac{q_{12} \bar{c}_{2}}{\left(1+q_{11} c_{1}\right)\left(1+q_{22} c_{2}\right)-q_{12} q_{21} c_{1} c_{2}} \\
\bar{h}_{21} & =\frac{q_{21} \bar{c}_{1}}{\left(1+q_{11} c_{1}\right)\left(1+q_{22} c_{2}\right)-q_{12} q_{21} c_{1} c_{2}} \\
\bar{h}_{22} & =\frac{q_{22} \bar{c}_{2}+\left(q_{11} q_{22}-q_{12} q_{21}\right) c_{1} \bar{c}_{2}}{\left(1+q_{11} c_{1}\right)\left(1+q_{22} c_{2}\right)-q_{12} q_{21} c_{1} c_{2}}
\end{aligned}
$$

The elements in $\bar{H}$ can be simplified by the following argument. The closed-loop bandwidth $\omega_{b}$ is limited by the right half-plane zeros of $G$. If there is a single zero in $z>0$, which is approximately equally distributed between the two loops, then the bandwidth must be less than $z \mathrm{rad} / \mathrm{s}$. It is seldom possible to achieve this high bandwidth with decoupled PID control, due to the restricted freedom in the implementation. Therefore, we pay our attention to less demanding control problems, where we do not want to push the performance to the limit. It is then natural to assume that the multivariable zeros of $G$ is not within the bandwidth of the closed-loop system. Recall that the zeros of $G$ is given by the solutions to the equation

$$
\operatorname{det} G(s)=g_{11}(s) g_{22}(s)-g_{12}(s) g_{21}(s)=0
$$

If we assume that $|\operatorname{det} G(s)| \gg 0$ for all $|s|<\omega_{b}$, then it follows from $\operatorname{det} Q(s)=\operatorname{det} G(s) \operatorname{det} D$ that $\left|q_{11}(s) q_{22}(s)\right| \gg\left|q_{12}(s) q_{21}(s)\right|$, since $\operatorname{det} Q(s)$ is a meromorphic function and $\operatorname{det} Q(0)=1$. The denominator of the elements $h_{i j}$ can then be approximated by $\left(1+q_{11} c_{1}\right)\left(1+q_{22} c_{2}\right)$. The numerator of $\bar{h}_{11}$ is approximated by $q_{11} \bar{c}_{1}+q_{11} q_{22} \bar{c}_{1} c_{2}=q_{11} \bar{c}_{1}\left(1+q_{22} c_{2}\right)$ and the numerator of $\bar{h}_{22}$ is approximated by $q_{22} \bar{c}_{2}+$ $q_{11} q_{22} c_{1} \bar{c}_{2}=q_{22} \bar{c}_{2}\left(1+q_{11} c_{1}\right)$. The matrix $\bar{H}$ is then approximated by

$$
H=\left(\begin{array}{cc}
\frac{q_{11} \bar{c}_{1}}{1+q_{11} c_{1}} & \frac{q_{12} \bar{c}_{2}}{\left(1+q_{11} c_{1}\right)\left(1+q_{22} c_{2}\right)} \\
\frac{q_{21} \bar{c}_{1}}{\left(1+q_{11} c_{1}\right)\left(1+q_{22} c_{2}\right)} & \frac{q_{22} c_{2}}{1+q_{22} c_{2}}
\end{array}\right)
$$

The structure of the diagonal elements of $H$ is the same as for SISO control design. Therefore, existing techniques [2] can be used for initial suggestions of how to design the decentralized controllers. Sometimes they have to be modified due to the interaction. The offdiagonal elements of $H$ tell us what interaction two SISO designs will lead to. Next, we exploit $H$ to come up with a systematic design procedure for decoupled PI control.

\section{Interaction Indices}

Before we discuss the actual design of the PI controllers $c_{i}$ and $\bar{c}_{i}$, let us elaborate further on the off-diagonal 
elements of $H$ :

$$
\begin{aligned}
& h_{12}=\frac{q_{12} \bar{c}_{2}}{\left(1+q_{11} c_{1}\right)\left(1+q_{22} c_{2}\right)} \\
& h_{21}=\frac{q_{21} \bar{c}_{1}}{\left(1+q_{11} c_{1}\right)\left(1+q_{22} c_{2}\right)}
\end{aligned}
$$

Due to the integral action in the controllers, the interaction is small at low frequencies. To estimate the maximum of the interaction, we observe that

$$
h_{12}=q_{12} \bar{c}_{2} S_{1} S_{2}, \quad h_{21}=q_{21} \bar{c}_{1} S_{1} S_{2}
$$

where $S_{1}=\left(1+q_{11} c_{1}\right)^{-1}$ and $S_{2}=\left(1+q_{22} c_{2}\right)^{-1}$ are the sensitivity for loop one and two, respectively, if the interaction is neglected. Upper bounds of the interaction terms are thus given by

$$
\begin{aligned}
\left|h_{12}(i \omega)\right| & \leq\left|q_{12} \bar{c}_{2}\right| M_{s 1} M_{s 2} \\
\left|h_{21}(i \omega)\right| & \leq\left|q_{21} \bar{c}_{2}\right| M_{s 1} M_{s 2}
\end{aligned}
$$

where $M_{s 1}$ and $M_{s 2}$ are the maximum sensitivity for the individual loops. For low frequencies, we have

$$
q_{12}(s) \approx \kappa_{12} s, \quad q_{21}(s) \approx \kappa_{21} s
$$

and

$$
\bar{c}_{1} \approx k_{I 1} / s, \quad \bar{c}_{2} \approx k_{I 2} / s
$$

For low frequencies, we thus find that $q_{12} \bar{c}_{2}$ and $q_{21} \bar{c}_{1}$ are constant. It is therefore natural to introduce the interaction indices

$$
\kappa_{1}=\left|\kappa_{12} k_{I 2}\right| M_{s 1} M_{s 2}, \quad \kappa_{2}=\left|\kappa_{21} k_{I 1}\right| M_{s 1} M_{s 2}
$$

The indices $\kappa_{1}$ and $\kappa_{2}$ describe the interaction of the second loop on the first and vice versa. Note that the indices are products of two terms: one depends on the system and the other is simply the integral gain of the corresponding PI controller. Interaction can thus be reduced by reducing the controller gains. We can ensure that the interaction is small by imposing a bound on the controller gains. This is natural because a small $k_{I 2}$ gives less coupling from the responses in the second loop and a large $k_{I 1}$ attenuate disturbances coming from the second loop. Note that $M_{s 1}$ and $M_{s 2}$ depend on $k_{I 1}$ and $k_{I 2}$, respectively. There is thus a trade-off between $\kappa_{1}$ and $\kappa_{2}$ : if $\left|\kappa_{12}\right|$ and $\left|\kappa_{21}\right|$ are of similar size, then both $\kappa_{1}$ and $\kappa_{2}$ cannot be made small by choosing appropriate integral gains. Either one of them can be made small while keeping the other larger, or both of them have to be kept at a medium level. Which approach to choose, follows from practical considerations of the importance of the individual control loops.

Another convenient measure to be used in the control design is the frequencies at which the interactions $\left|h_{12}(i \omega)\right|$ and $\left|h_{12}(i \omega)\right|$ attain their maxima. These frequencies will determine the bandwidth of the closedloop system. It is possible to make the following simplifications in order to derive estimates of the frequencies. Since the controllers have integral action, we have for small $s$

$$
h_{12}(s) \approx \frac{\kappa_{12} s^{2}}{k_{I 1}}, \quad h_{21}(s) \approx \frac{\kappa_{21} s^{2}}{k_{I 2}}
$$

For large $s$, the assumption $\left|q_{11}(s) q_{22}(s)\right| \gg$ $\left|q_{12}(s) q_{21}(s)\right|$ used to derive $H$ is still reasonable, because this means that the diagonal elements of $G$ is dominating at high frequencies. Hence, the transfer function $H$ describes the relation between $Y_{r}$ and $Y$ also for large $s$. This leads to that

$$
h_{12}(s) \approx \frac{k_{I 2}}{s^{d_{1}+1}}, \quad h_{21}(s) \approx \frac{k_{I 1}}{s^{d_{2}+1}}
$$

for large $s$, where $d_{1}$ is the pole excess of $q_{12}(s)$ and $d_{2}$ is the pole excess of $q_{21}(s)$. Note that $d_{1}$ and $d_{2}$ are uniquely defined by $G$, and do not depend on the controllers. It follows from (3) and (4) that $\left|h_{12}(i \omega)\right|$ and $\left|h_{21}(i \omega)\right|$ will have maxima. The location of the maximum of $\left|h_{12}(i \omega)\right|$ can, by using (3) and (4), be approximated by the solution of the equation

$$
\frac{\left|\kappa_{12}\right| \omega^{2}}{\left|k_{I 1}\right|}=\frac{\left|k_{I 2}\right|}{\omega^{d_{1}+1}}
$$

The peaks of the diagonal terms $\left|h_{12}(i \omega)\right|$ and $\left|h_{21}(i \omega)\right|$ are thus approximately located at

$$
\omega_{12}=\sqrt[d_{1}+3]{\left|\frac{k_{I 1} k_{I 2}}{\kappa_{12}}\right|}, \quad \omega_{21}=\sqrt[d_{2}+3]{\left|\frac{k_{I 1} k_{I 2}}{\kappa_{21}}\right|}
$$

If the pole excesses of $q_{12}$ and $q_{21}$ are equal to one, then $d_{1}=d_{2}=1$, so that

$$
\omega_{12}=\sqrt[4]{\left|\frac{k_{I 1} k_{I 2}}{\kappa_{12}}\right|}, \quad \omega_{21}=\sqrt[4]{\left|\frac{k_{I 1} k_{I 2}}{\kappa_{21}}\right|}
$$

The interaction indices (2) are derived for PI controllers with set-point weighting $b_{1}=b_{2}=0$. The interactions increase significantly if the controller do not have setpoint weighting. If $b_{1}=b_{2}=1$ then the interaction measures become

$$
\begin{aligned}
& \kappa_{1}=\left|\kappa_{12}\right|\left(\left|k_{P 2}\right| \omega+\left|k_{I 2}\right|\right) M_{s 1} M_{s 2} \\
& \kappa_{2}=\left|\kappa_{21}\right|\left(\left|k_{P 1}\right| \omega+\left|k_{I 1}\right|\right) M_{s 1} M_{s 2}
\end{aligned}
$$

Finally, it should be remarked that the estimate (2) can be too conservative if there is a significant difference in the bandwidths of the loops.

\section{Design of Decentralized Controllers}

To find the decentralized PID controller, we consider the diagonal terms of the transfer function $Q(s)$. By construction we know that the static gain of all diagonal elements are unity. Standard methods can be used for the design of PI or PID controller for each transfer function $q_{k k}(s)$. One possibility is to use an 


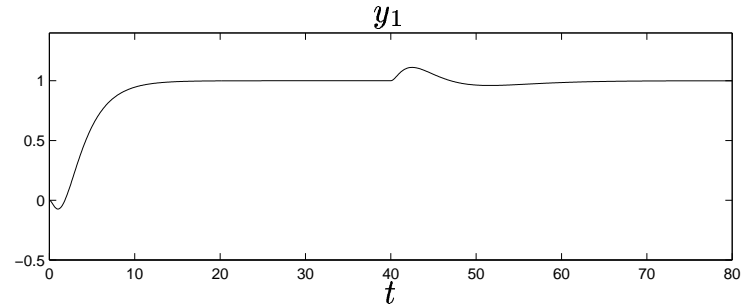

$y_{2}$

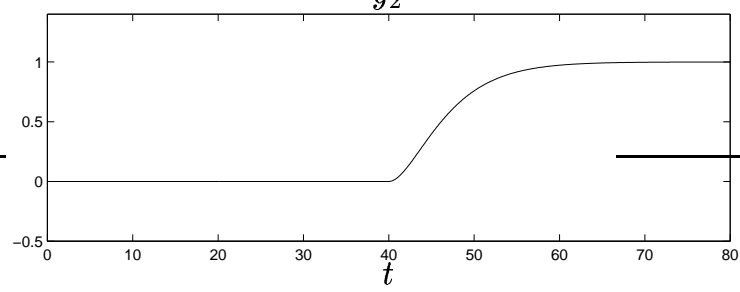

Figure 1: Simulation of the design method applied to Rosenbrock's system. The figure shows the response of the outputs to steps in the command signals. The PI controllers have set-point weighting $b_{1}=b_{2}=0$.

optimization technique which minimizes integral gain subject to a robustness constraint. This is discussed for PI controllers in [3] and for PID controllers in [10]. Such a method will give controllers which are optimized with respect to rejection of load disturbances. The design methods will give integral gains $k_{I k}^{0}>0$, which could be used if there were no interactions. The ratios $\lambda_{k}=k_{I k} / k_{I k}^{0}$ are thus measures of performance losses due to the interaction. If $\lambda_{k} \geq 1$ there are no performance losses and the limitations on performance are essentially given by the loop dynamics. If $0 \leq \lambda_{k}<1$ it is necessary to detune the controllers. In this case we need methods to design PID controllers with specifications on the closed-loop bandwidth. There are many methods which can be used for this purpose. Two simple techniques for design of PID controllers are the direct pole-placement design based on reduced-order models and the dominant pole design, see [2] and [11].

\section{Examples}

The control design method is in this section illustrated on two examples from the literature.

\section{Rosenbrock's System}

The process

$$
G(s)=\left(\begin{array}{cc}
\frac{1}{s+1} & \frac{2}{s+3} \\
\frac{1}{s+1} & \frac{1}{s+1}
\end{array}\right)
$$

was originally proposed by Rosenbrock [13]. It is an example of a system that looks very easy to control, but which has fundamental limitations because it has
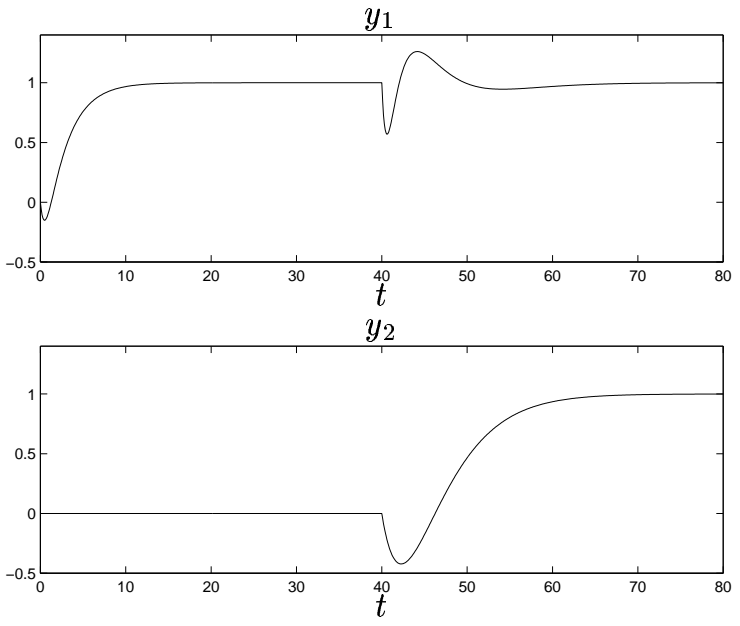

Figure 2: Simulation of controllers without set-point weighting. The simulation is identical to Figure 1 but the controllers have $b_{1}=b_{2}=1$.

a zero $s=1$ in the right half-plane. If we introduce static decoupling, the compensated transfer function becomes

$$
Q(s)=\left(\begin{array}{cc}
\frac{3(1-s)}{(s+1)(s+3)} & \frac{4 s}{(s+1)(s+3)} \\
0 & \frac{1}{s+1}
\end{array}\right)
$$

The interaction is given by $\kappa_{12}=4 / 3$ and $\kappa_{21}=0$. Since $\kappa_{21}=0$, interaction gives no performance limitations for the second loop. There are however limitations because of the right half-plane zero at $s=1$. Designing a PI controller that maximizes integral gain subject to the constraints that the maximum sensitivity $M_{s 1}$ and the maximum complementary sensitivity $M_{p 1}$ are less than $\sqrt{2}$, gives $k_{P 1}=0.245$ and $k_{I 1}=0.248$, see [3]. Since $\kappa_{12}=4 / 3$ there are constraints on the design of the first loop because of the coupling. Requiring that the coupling $\kappa_{1}$ is less than 0.1 and the maximum sensitivity $M_{s 2}$ is less than $\sqrt{2}$, we find that the integral gain of the second loop $k_{I 2}$ must be less than $\kappa_{1} /\left(\kappa_{12} M_{s 1} M_{s 2}\right)=0.0750$. To design a PI controller, we use direct pole placement [2] based on the model $q_{22}(s)=(s+1)^{-1}$. This gives $k_{P 2}=2 \zeta \omega_{0}-1$ and $k_{I 2}=\omega_{0}^{2}$. Requiring that the integral gain is equal to 0.0750 , we find that $\omega_{0}=0.274$. With $\zeta=1$ the controller gains become $k_{P 2}=-0.452$ and $k_{I 2}=0.075$. Figure 1 shows simulations of set-point responses for the closed-loop system. The plots show the proposed design with set-point weighting $\left(b_{1}=b_{2}=0\right)$. A unit step in the set point of the first controller is applied at time $t=0$ and a step in the set point of the second controller is then applied at time $t=40$. Figure 2 shows the step responses for a controller without set-point weighting. The figure clearly indicates the advantage of set-point weighting for multivariable systems. The reason why there is such a large difference is that the control signal is much smoother with set-point weight- 

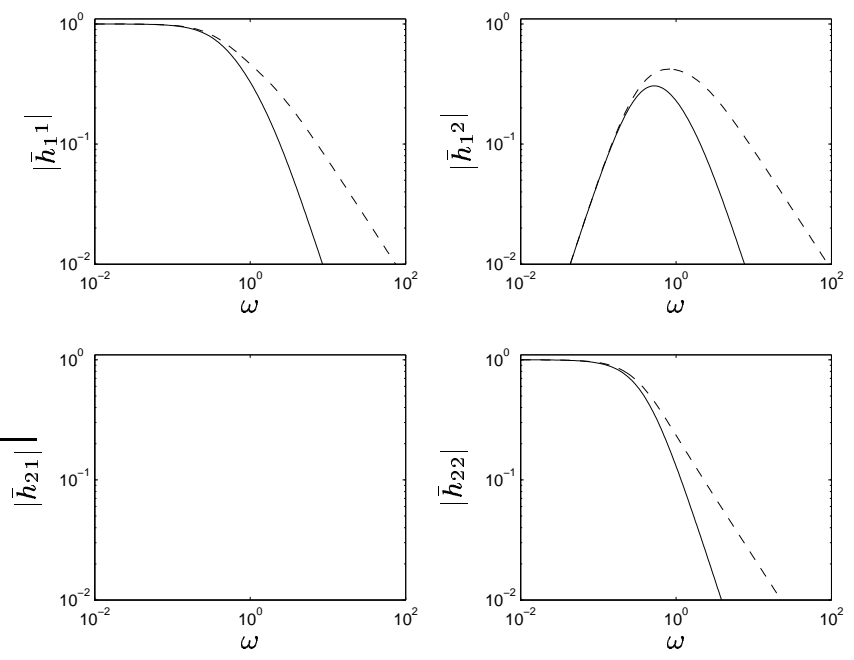

Figure 3: The frequency response of the closed-loop system with set-point weighting (solid) and without (dashed). Note that without set-point weighting the interaction $\left|\bar{h}_{12}(i \omega)\right|$ is larger and extends to higher frequencies.

ing.

The effect of set-point weighting is illustrated also in Figure 3, which shows the frequency response of the closed-loop system with (solid) and without (dashed) set-point weighting. The interaction increases considerably when no set-point weighting is applied. This agrees with the conclusion in previous section, compare the interaction indices with and without set-point weighting in Equations (2) and (6).

The maximum of the interaction $\left|\bar{h}_{12}(i \omega)\right|$ in Figure 3 is equal to 0.3 . This should be compared to the interaction index $\kappa_{1}=0.2$. The maximum is attained at $\omega=0.52$, while the estimate in (5) predicts 0.41 .

It is interesting to note that the bandwidth of the closed-loop system (as measured by the maximal singular value) is equal to $\omega_{b}=0.49 \mathrm{rad} / \mathrm{s}$, which is fairly close to the limitation imposed by the right half-plane zero at +1 .

\section{Wood-Berry's Binary Distillation Column}

The Wood-Berry binary distillation column plant [14] is a multivariable system that has been studied extensively. The process has the transfer function

$$
G(s)=\left(\begin{array}{ll}
\frac{12.8 e^{-s}}{16.7 s+1} & \frac{-18.9 e^{-3 s}}{21.0 s+1} \\
\frac{6.60 e^{-7 s}}{10.9 s+1} & \frac{-19.4 e^{-3 s}}{14.4 s+1}
\end{array}\right)
$$

The transfer function of the statically compensated system is

$$
Q(s)=\left(\begin{array}{cc}
\frac{12.8 e^{-s}}{16.7 s+1} & \frac{-18.9 e^{-3 s}}{21.0 s+1} \\
\frac{6.60 e^{-7 s}}{10.9 s+1} & \frac{-19.4 e^{-3 s}}{14.4 s+1}
\end{array}\right)\left(\begin{array}{cc}
0.1570 & -0.1529 \\
0.0534 & -0.1036
\end{array}\right)
$$

A series expansion for small $s$ gives

$$
Q(s) \approx\left(\begin{array}{cc}
1-11.7 s & -12.31 s \\
-0.5138 s & 1-17.3 s
\end{array}\right)
$$

The interaction is thus given by $\kappa_{12}=-12.31$ and $\kappa_{21}=-0.5138$. The time constants of the reducedorder models are $T_{1}=11.7$ and $T_{2}=17.3$. Let the desired maximum sensitivity for the individual loops be equal to $M_{s 1}=M_{s 2}=\sqrt{2}$. Requiring that the interaction indices $\kappa_{1}$ and $\kappa_{2}$ should both be less than 0.20 , the integral gains should be $k_{I 1}<0.19$ and $k_{I 2}<0.0081$. This gives the corresponding crossover frequencies

$$
\begin{aligned}
& \omega_{01}<\sqrt{k_{I 1} / T_{1}}=\sqrt{0.19 / 11.7}=0.12 \\
& \omega_{02}<\sqrt{k_{I 2} / T_{2}}=\sqrt{0.0081 / 17.3}=0.022
\end{aligned}
$$

We have $\omega_{01} L=0.36$ and $\omega_{02} L=0.066$. The bandwidths are thus well below the limitations $\omega L<0.7$ imposed by the the largest time delay of the system $(L=3)$, see [1].

With these frequencies and the time delays present in the system, it is quite reasonable to approximate the time delays with first order lags. The model reduction should thus work reasonably well in this case. Choosing $\zeta_{1}=\zeta_{2}=\zeta=0.707$ we find that the proportional gains are

$$
\begin{aligned}
& k_{P 1}=2 \zeta \omega_{01}-1=1.1338 \\
& k_{P 2}=2 \zeta \omega_{02}-1=-0.4699
\end{aligned}
$$

In Figure 4 we show the step responses from set point to process output for the system. Analyzing the figure we find that the response in $y_{1}$ caused by a step in the set point $y_{r 2}$ is quite small. It thus seems possible to increase the bandwidth in the second loop. After some experimentation we found that a reasonable value is $\omega_{02}=0.1$, which gives that the controller parameters for the second loop become $k_{P 2}=0.7$ and $k_{I 2}=0.123$. The time responses are shown in Figure 5. A comparison with Figure 4 shows that the response speed of the second loop has been increased considerably without increasing the interaction too much. This also shows that the method lends itself very well to tuning. The step responses in Figure 5 compare favorably with designs obtained by other methods.

\section{Conclusions}

The suggested control scheme is a quite simple multivariable controller. However, it handles a large number of practical control problems, although it can be implemented with regular PID controllers together with a proportional controller $D$. It is desirable to have efficient design methods for decoupled PID control. Such a method has been developed in the paper, under the 
$y_{1}$
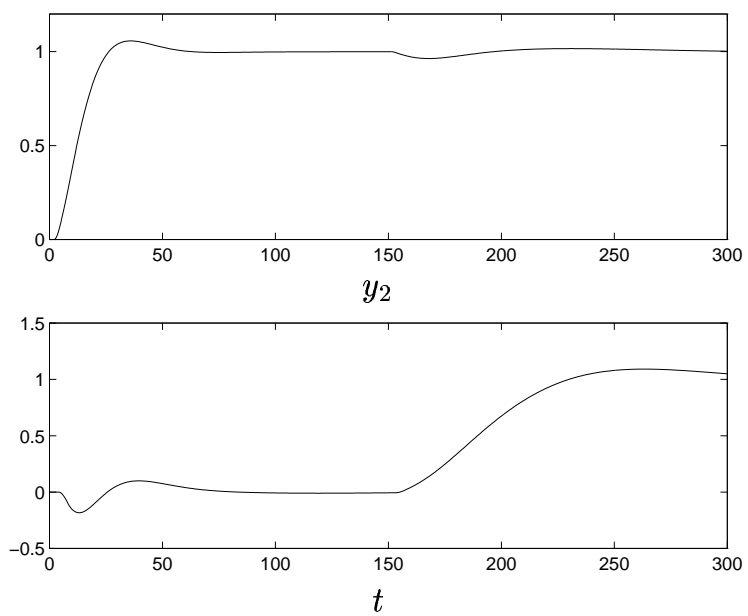

Figure 4: Simulation of the design method applied to the Wood-Berry distillation column. The figure shows the response of the outputs to steps in the command signals.

assumption that the interaction is not too severe. Possible detuning of the PID controllers was quantified via the interaction indices $\kappa_{1}$ and $\kappa_{2}$. An important remark made in the paper was that set-point weighting is necessary for PID controllers in multivariable systems. The interaction between the control loops can be reduced considerably if set-point weighting $b_{1}=b_{2}=0$ is used.

Other methods for doing detuning for multivariable PID control includes Niederlinski's heuristic design [9]. Note that the interaction indices $\kappa_{1}$ and $\kappa_{2}$ depend on the controller, which is for instance not the case for the widely used interaction measure RGA by Bristol [4].

\section{References}

[1] K. J. Åström. Limitations on control system performance. European Journal on Control, 6:2-20, 2000.

[2] K. J. Åström and T. Hägglund. PID Controllers: Theory, Design, and Tuning. Instrument Society of America, Research Triangle Park, NC, 1995.

[3] K. J. Åström, H. Panagopoulos, and T. Hägglund. Design of PI controllers based on non-convex optimization. Automatica, 34(5):585-601, 1998.

[4] E. Bristol. On a new measure of interaction for multivariable process control. IEEE Trans. Autom. Control, 11:133, 1966.

[5] E. F. Camacho and C. Bordons. Model Predictive Control. Springer, London, 1999.

[6] Y. Lee, S. Park, and J. H. Lee. On interfacing model predictive controllers with low-level loops. In Preprints. 14th World Congress of IFAC, volume N, pages 313-318, Beijing, China, 1999. $y_{1}$
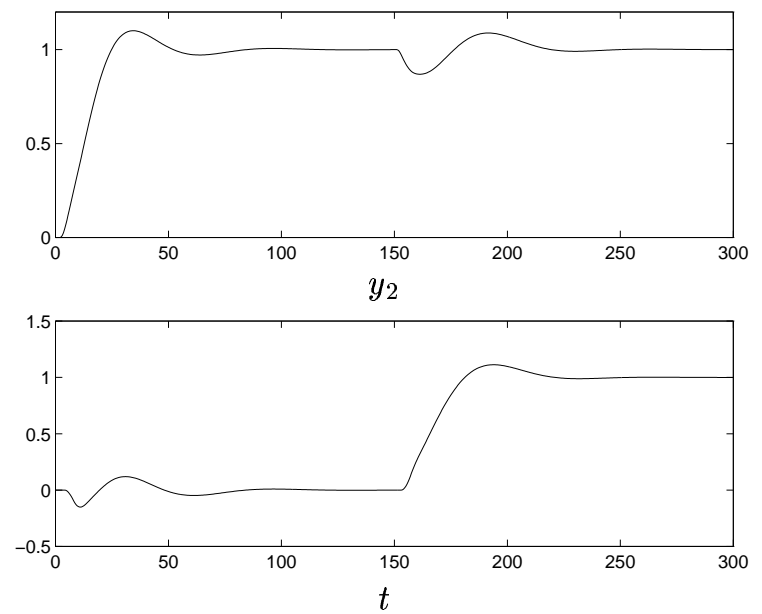

Figure 5: Simulation of the design method applied to the Wood-Berry distillation column. The bandwidth of the second loop has been increased to $\omega_{02}=0.1$.

[7] W. L. Luyben. Process modeling, simulation, and control for chemical engineers. McGraw-Hill, New York, 1990.

[8] J. M. Maciejowski. Predictive Control with Constraints. Pearson Education, 2000.

[9] A. Niederlinski. A heuristic approach to the design of linear multivariable interacting control systems. Automatica, 7:691-701, 1971.

[10] H. Panagopoulos. PID-Control. Design, Extension, Application. PhD thesis, Dept. of Automatic Control, Lund University, Sweden, 2000.

[11] P. Persson and K. J. Åström. Dominant pole design - a unified view of PID controller tuning. In Preprints 4th IFAC Symposium on Adaptive Systems in Control and Signal Processing, pages 127-132, Grenoble, France 1992.

[12] S. J. Qin and T. A. Badgwell. An overview of industrial model predictive control technology. In J. C. Kantor, editor, Conference on Chemical Process Control CPC V, pages 232-256, London, 1996. CACHE, Elsevier.

[13] H. H. Rosenbrock. State-Space and Multivariable Theory. Nelson, London, U.K., 1970.

[14] R. K. Wood and M. W. Berry. Terminal composition control of a binary distillation column. Chem. Engng. Sci., 28:1707-1717, 1973. 